\title{
Optimisation in vitro de l'efficacité des biopesticides dans la lutte contre les principaux ravageurs du niébé par la méthode des surfaces de réponse
}

\author{
A. GOUGBE SEMAKO ${ }^{1,2,3}$, K. ABOUDOU ${ }^{1}$, K. O. CHATIGRE ${ }^{3}$, \\ M. S. P. NOUKPOZOUNKOU ${ }^{1}$ et M. M. SOUMANOU ${ }^{1 *}$ \\ ${ }^{1}$ Université d'Abomey-Calavi, Ecole Polytechnique d'Abomey-Calavi, Département de Génie de Technologie \\ Alimentaire, Unité de Recherche en Génie Enzymatique et Alimentaire, Laboratoire d'Etude et de Recherche \\ en Chimie Appliquée (LERCA) 01 BP 2009 Cotonou 01, Bénin. \\ ${ }^{2}$ Université Félix HOUPHOUET BOIGNY, Centre d'Excellence Africain sur les \\ Changements Climatiques Biodiversité et Agriculture Durable (CEA-CCBAD), Tél : +225073051 55 ; \\ Abidjan, Côte d'Ivoire. \\ ${ }^{3}$ Université Félix HOUPHOUET BOIGNY, Unité de Formation pour la Recherche en \\ Bioscience, Laboratoire de Biochimie et Science des Aliments, 22 BP 582 Abidjan 22, Tel: +225 22444473 , \\ Côte d'Ivoire. \\ *Auteur correspondant ; E-mail: msoumanoufr@yahoo.fr
}

Received: 27-10-2020 Accepted: 20-02-2021 $\quad$ Published: 28-02-2021

\section{RESUME}

La culture du niébé requiert de nos jours, une attention particulière à cause de sa sensibilité aux insectes ravageurs. La présente étude vise à déterminer, au laboratoire, les doses optimales de la combinaison des biopesticides (Topbio et virus MaviMNPV) à utiliser dans la lutte contre les principaux ravageurs du niébé notamment, Maruca vitrata, Aphis craccivora, et Megalurothrips sjostedti. A cet effet, les insectes ont été inoculés avec différentes doses des combinaisons de Topbio et du virus MaviMNPV variant respectivement de $660 \mathrm{ml}$ à $1000 \mathrm{ml}$ et de $70 \mathrm{ml}$ à $106 \mathrm{ml}$ en utilisant la méthode des surfaces de réponses. La mortalité et la survie des insectes ont été évaluées et les doses optimales de la combinaison des biopesticides ont été déterminées. Les résultats ont montré que les effets linéaires et/ou quadratiques de la combinaison des biopesticides influencent significativement les taux de mortalité, d'émergence des larves et de mortalité des chrysalides. Ces taux ont varié de 63,33 à $85 \%$, de 12,5 à $37,5 \%$, et de 44,44 à $87,5 \%$ respectivement pour les taux de mortalité, d'émergence des larves et de mortalité des chrysalides de Maruca vitrata. Les taux de mortalité des formes adultes de Aphis craccivora, et Megalurothrips sjostedti, ont varié respectivement de 88,33 à $95 \%$ et de 85 à $95 \%$. L'efficacité optimale des biopesticides est obtenue pour $1000 \mathrm{ml}$ de Topbio et $106 \mathrm{ml}$ du virus MaviMNPV avec un taux de désirabilité de $85 \%$.

(C) 2021 International Formulae Group. All rights reserved.

Mots clés : Topbio, virus MaviMNPV, ravageurs, taux de mortalité, taux d'émergence. 


\title{
In vitro optimization of the effectiveness of biopesticides in the control of the main pests of cowpea by using response surface methodology
}

\begin{abstract}
Nowadays, cowpea cultivation requires a special attention due to its sensibility to pest insects. The present study aimed at determining, in laboratory conditions, the optimal doses of the combination of biopesticides (Topbio and MaviMNPV virus) used in the control of the main pre-harvest pests of cowpea especially Maruca vitrata, Aphis craccivora, et Megalurothrips sjostedti. For this purpose, the insects were inoculated with the doses of the combinations of Topbio and the MaviMNPV virus varying respectively from 660 $\mathrm{ml}$ to $1000 \mathrm{ml}$ and from $70 \mathrm{ml}$ to $106 \mathrm{ml}$, respectively using the response surfaces method. The mortality and survival of the larvae were assessed and the optimal doses of the combination of biopesticides were determined. The results showed that the linear and / or quadratic effects of the combination of biopesticides significantly influenced the mortality, larval emergence and pupa mortality rates. These rates varied from 63.33 to $85 \%$, from 12.5 to $37.5 \%$, and from 44.44 to $87.5 \%$ for the mortality, emergence of larvae and mortality rates of pupae, respectively. Maruca vitrata. The mortality rates of adult of Aphis craccivora, and Megalurothrips sjostedti, ranged from 88.33 to $95 \%$ and 85 to $95 \%$, respectively. Optimal efficacy of biopesticides is obtained for 1000 $\mathrm{ml}$ of Topbio and $106 \mathrm{ml}$ of MaviMNPV virus with a desirability rate of $85 \%$.
\end{abstract}

(C) 2021 International Formulae Group. All rights reserved.

Keywords: Topbio, MaviMNPV virus, pests, mortality rate, emergence rate.

\section{INTRODUCTION}

Le niébé (Vigna unguiculata L. Walp) est l'une des plus importantes légumineuses cultivées et consommées en Afrique (Kadri et al., 2013 ; Boukar et al., 2019). Sa contribution à la réduction de la pauvreté et à l'amélioration de la sécurité alimentaire est considérable (Sanou et al., 2016). En effet, il a une importance capitale dans le régime alimentaire et possède une forte teneur en protéines (20 à $35 \%$ de poids sec) et particulièrement en acides aminés essentiels (El-Jasser, 2011). Malgré sa déficience en méthionine et en cystéine, il représente une denrée de base en Afrique subsaharienne et surtout en période de sécheresse, lorsque celles qui sont principales viennent à manquer. Ses besoins en azote sont peu élevés et ses racines sont munies de nodosités peuplées de bactéries (Rhizobiums) qui contribuent à la fixation de l'azote atmosphérique. En dépit des nombreuses vertus que regorgent cette précieuse légumineuse, sa culture est confrontée à de sérieuses contraintes dont principalement l'attaque des insectes ravageurs (Aphis craccivora, Megalurothrips sjostedti, Maruca vitrata et les Clavigralla tomentosicollis etc) depuis la culture jusqu'en stocks (Traore et al., 2019). Face à cette situation, l'utilisation des pesticides de synthèse représente la première solution à portée de mains. Cependant face aux nombreux dégâts que l'utilisation abusive des pesticides de synthèse chimiques occasionne sur l'homme, les animaux et l'environnement il urge donc de porter les regards vers des solutions simples et respectueuses de l'homme et de la biodiversité. Ce fut alors l'avènement des méthodes de lutte biologique (origine végétale ou microbienne) basées sur l'utilisation des extraits de plantes (Kpoviessi et al., 2017 ; Adjoub et al., 2019 ; Traore et al., 2019), des bactéries (Valda et al., 2003), des virus (Sokame et al., 2015) et des champignons (Mehinto et al., 2014) ou de leur sous-produits (Reddy et al., 2008). A cause de leur grande disponibilité dans le contexte Ouest-africain, une attention est de plus en plus portée sur les extraits de plantes et les biopesticides à base de virus (Ben et al., 2005 ; Yarou et al., ; 2017 ; Abdoulaye et al., 2018; Abdourahamane Harouna et al., 2019). Ainsi, les travaux réalisés ces dernières années ont montré que le 
virus MaviMNPV, extrait des larves de Maruca vitrata, extermine spécifiquement le ravageur le plus redoutable du niébé (Maruca vitrata) (Ekesi, 1999 ; Sokame et al., 2015). En effet, après ingestion du virus par les larves de Maruca vitrata à travers l'aliment contaminé, ce dernier s'attaque à l'hémolymphe, aux tissus adipeux et à l'intestin moyen de l'insecte (Maruca vitrata). Ils liquéfient les corps gras et entraînent une turgescence de l'insecte suivi de sa mort (Hounhouigan et al., 2014). Cette spécificité des baculovirus constitue un atout, mais implique en contrepartie l'utilisation de plusieurs agents pathogènes pour traiter une même culture attaquée par une gamme d'insectes. Cependant, l'extrait de neem (l'azadirachtine), le biopesticide d'origine végétale le plus utilisé a présenté selon plusieurs études (Camarda et al., 2018 ; Muhammad et al., 2018), un effet très significatif sur plus de 400 espèces d'insectes ravageurs de culture dont Aphis craccivora et Megalurothrips sjostedti piqueurs-suceurs vivant à l'intérieur des fleurs de niébé et s'alimentant de sa sève (Egho, 2011). Le principe actif (l'azadirachtine) de ce dernier agit même à de faibles doses en inhibant le comportement alimentaire chez ces insectes, à travers le manque de stimulation des cellules du tube digestif (Mouffok et al., 2007 ; Lokanadhan et al., 2012). Ce qui induit un retard dans la croissance et une perte de poids suivie le plus souvent de la mort de l'insecte. Par ailleurs, les travaux réalisés sur l'utilisation des biopesticides dans la lutte contre les ravageurs ont révélé que les doses des biopesticides utilisées influencent non seulement la productivité, mais également la qualité marchande des graines du niébé. La combinaison des dérivées du neem et du virus MaviMNPV, s'avère plus efficace que ces biopesticides utilisés individuellement à des concentrations variant de 12 à $50 \%$ d'huile de neem (Sokame et al., 2015 ; Kindozandji et al., 2020). Il convient donc de trouver les doses adéquates des combinaisons des biopesticides pouvant aboutir à une meilleure productivité.
Le présent travail vise à optimiser les doses des combinaisons de Topbio (l'azadirachtine) et du virus MaviMNPV.

\section{MATERIEL ET METHODES Matériel}

Le matériel végétal utilisé était constitué des graines de la variété locale de niébé communément appelée «TAWA » produites à l'Institut International d'Agriculture Tropicale (IITA-Bénin). TopBio dont le principe actif principal est l'azadirachtine est un biopesticide $100 \%$ naturel et dont la composition chimique est caractérisée par la présence de nimbin, de azadirachtine, de citronellol, de citronellal, et de géraniol, est un biopesticide $100 \%$ naturel et couramment utilisé dans la lutte contre les insectes ravageurs. La souche du virus de la polyédrose nucléaire NPV (MaviMNPV), isolé des larves de $M$. vitrata a été fournie par le laboratoire de phytopathologie de l'IITABénin. L'extrait de virus utilisé pour les essais est obtenu après inoculation de larves saines de $M$. vitrata dans une solution mère obtenue à partir de la poudre de larves virosées originaires de Taïwan et introduite au Bénin en 2006 par l'IITA. Cette solution est conservée au réfrigérateur à $4{ }^{\circ} \mathrm{C}$ afin de maintenir sa virulence initiale. Les principaux insectes ravageurs utilisés notamment Aphis craccivora, Megalurothrips sjostedti, et Maruca vitrata ont été fournis par le laboratoire d'entomologie de l'IITA-Bénin.

\section{Méthodes}

Site d'étude

Les travaux ont été conduits au laboratoire d'entomologie de la station de l'Institut International d'Agriculture Tropicale (IITA-Bénin) au Bénin où la température et l'humidité relative moyennes étaient respectivement de $26 \pm 1{ }^{\circ} \mathrm{C}$ et de $70 \pm 1 \%$. L'IITA-Bénin est situé à Togoudo $\left(06^{\circ} 2155^{\circ} \mathrm{N}\right.$, $02^{\circ} 2550$ 'E) dans la commune d'AbomeyCalavi, département de l'Atlantique, au Sud du Bénin. Il est situé à $12 \mathrm{~km}$ au Nord-Ouest de 
Cotonou (capitale économique de la République du Bénin) et à 2,5 km environ de la voie inter État Cotonou-Bohicon avec une altitude de $15 \mathrm{~m}$.

\section{Dispositif expérimental}

Les essais ont été réalisés selon la méthode de Mikpon (2017). Pour chaque type de ravageur, vingt insectes ont été utilisés dans un dispositif en bloc complètement aléatoire avec trois répétitions par combinaison, soit soixante insectes par combinaison de biopesticides. Pour chaque type d'insecte, 780 individus ont été utilisés, à raison de 60 insectes x 13 combinaisons de biopesticides. Après isolement, les insectes ont été introduits individuellement dans de petites boîtes de dimensions $4 \times 2 \times 4 \mathrm{~cm}$, avec des couvercles perforés et laissés ensuite affamés pendant $6 \mathrm{~h}$. Cela permet d'éliminer les insectes faibles ou blessés pendant l'isolement, afin de s'assurer que les futurs insectes morts seraient effectivement morts du traitement appliqué. Pendant ce temps, les graines de niébé prégermées de $48 \mathrm{~h}$ (pour nourrir Maruca vitrata), et les pédoncules ou gousses fraîches découpées en de petits morceaux et dont les extrémités sont bouchées par la paraffine (pour nourrir Megalurothrips sjostedti et Aphis craccivora), sont trempées dans chacune des solutions des différentes combinaisons de biopesticides, pendant 10 minutes. Le traitement témoin est constitué de 60 insectes traités séparément avec du niébé prégermé ou pédoncule ou gousse trempé, selon le ravageur, dans une solution de tween $0.1 \%$ stérilisée. Les organes végétaux trempés, ont été ensuite retirés et séchés par ventilation dans les conditions ambiantes pendant 15 minutes, et mis dans chaque boîte, à raison de deux organes par boîte contenant un insecte ou larve affamée depuis 6h. Les boîtes ont été ensuite déposées dans des plateaux au laboratoire.

\section{Plan expérimental}

La méthode des surfaces de réponse (RSM), à travers un plan composite centré, a été utilisée pour optimiser les doses des biopesticides (Topbio et virus MaviMNPV)
(Kek et al., 2014). Les doses de Topbio et virus MaviMNPV ont variées respectivement de 660 $\mathrm{ml}$ à $1000 \mathrm{ml}$ et de $70.10^{9}$ conidies $/ \mathrm{ml}$ à $106.10^{9}$ conidies/ml. Le plan expérimental a généré 13 essais (Tableau 1), conduits suivant un dispositif en blocs complètement aléatoires, et incluant plusieurs facteurs. Il s'agit des espèces d'insectes (Megalurothrips sjostedti (Trybom), ravageurs floricoles; Maruca vitrata (Fabricius), foreur de fleurs et de gousses ; les pucerons, Aphis craccivora (Koch)) ; et les combinaisons à base de Topbio et du virus MaviMNPV

\section{Evaluation du taux de mortalité des insectes}

Le taux de mortalité a été évalué suivant la méthode décrite par Kumar et al. (2014). Vingt-quatre heures après l'inoculation, les insectes morts ont été retirés des boîtes d'inoculation et ceci quotidiennement jusqu'à la formation des chrysalides ou l'émergence des adultes. Les larves mortes sporulées, les chrysalides formées et les adultes émergés ont été également comptés. Le taux de mortalité des insectes (TM) a été ensuite calculé selon la formule (1) (Abdoulaye et al., 2018) suivante :

$T M(\%)=\frac{\text { Nbre d'insectes morts }}{\text { Nbre d'insectes total }} \times 100$

En réponse à ces traitements appliqués, le taux de mortalité des larves de Maruca vitrata à 3 jours après l'inoculation, et à 9 jours après l'inoculation; le taux de mortalité des Megalurothrips sjostedti à 3 jours après l'inoculation, et à 9 jours après l'inoculation ; le taux de mortalité des Aphis craccivora à 3 jours après l'inoculation et à 9 jours après l'inoculation; et les taux d'émergence et de mortalité des chrysalides de Maruca vitrata ont été évalués. Une équation de second degré a été définie à partir des expérimentations pour prédire l'évolution des différentes réponses en fonction des paramètres étudiés (Myers et Montgomery, 1995) suivant la formule ciaprès :

$$
\mathrm{Y}=\mathrm{b}_{0}+\mathrm{b}_{1} \mathrm{x}_{1}+\mathrm{b}_{2} \mathrm{x}_{2}+\mathrm{b}_{12} \mathrm{x}_{1} \mathrm{x}_{2}+\mathrm{b}_{11} \mathrm{x}_{1}^{2}+
$$
$\mathrm{b}_{22} \mathrm{x}_{2}^{2}(2)$ 
Avec : $\mathrm{Y}$ : réponse prédite $; b_{0}, b_{1}, b_{2}$, $b_{11}$, et $b_{22}$ : coefficients de l'équation, avec $b_{0}$ : constante; $b_{1}$ et $b_{2}$ : coefficients des termes linéaires; $b_{11}$ et $b_{22}$ : coefficients des termes quadratiques et $b_{12}$ : coefficients d'interaction. $\mathrm{x}_{1}$ et $\mathrm{x}_{2}$ : valeurs codées des variables indépendantes des doses du virus MaviMNPV et de Topbio respectivement. Les valeurs codées ont été obtenues par transformation de valeurs réelles en utilisant la relation suivante : $x i=\frac{X i-X 0 i}{\Delta \mathrm{X}}$

Avec Xi : valeur réelle de la variable : Xoi : valeur centrale de la variable et $\Delta \mathrm{X}$ l'incrément.

\section{Analyses statistiques}

Les données ont été traitées à l'aide du logiciel Minitab 16 (Minitab Inc., USA). Les courbes de surface de réponses ont été générées graphiquement pour évaluer l'influence des doses de biopesticides sur les paramètres étudiés. Les conditions optimales de la combinaison des biopesticides ont été obtenues et la fonction de désirabilité était utilisée pour déterminer les doses optimales de Topbio et de virus. Les valeurs des fonctions de désirabilité (d) sont comprises entre 0 et 1 . Les réponses obtenues expérimentalement ont été comparées aux valeurs prédites à partir du modèle optimisé en calculant le pourcentage d'erreur (PE) qui est inférieur à 10\%, qui indique un bon ajustement (Kek et al., 2014).

$P E(\%)=[($ mexp- mpre $) /$ mexp $)] \times 100$, avec mexp : valeur expérimentale et mpre : valeur prédite.

Tableau 1 : Paramètres du plan composite centré.

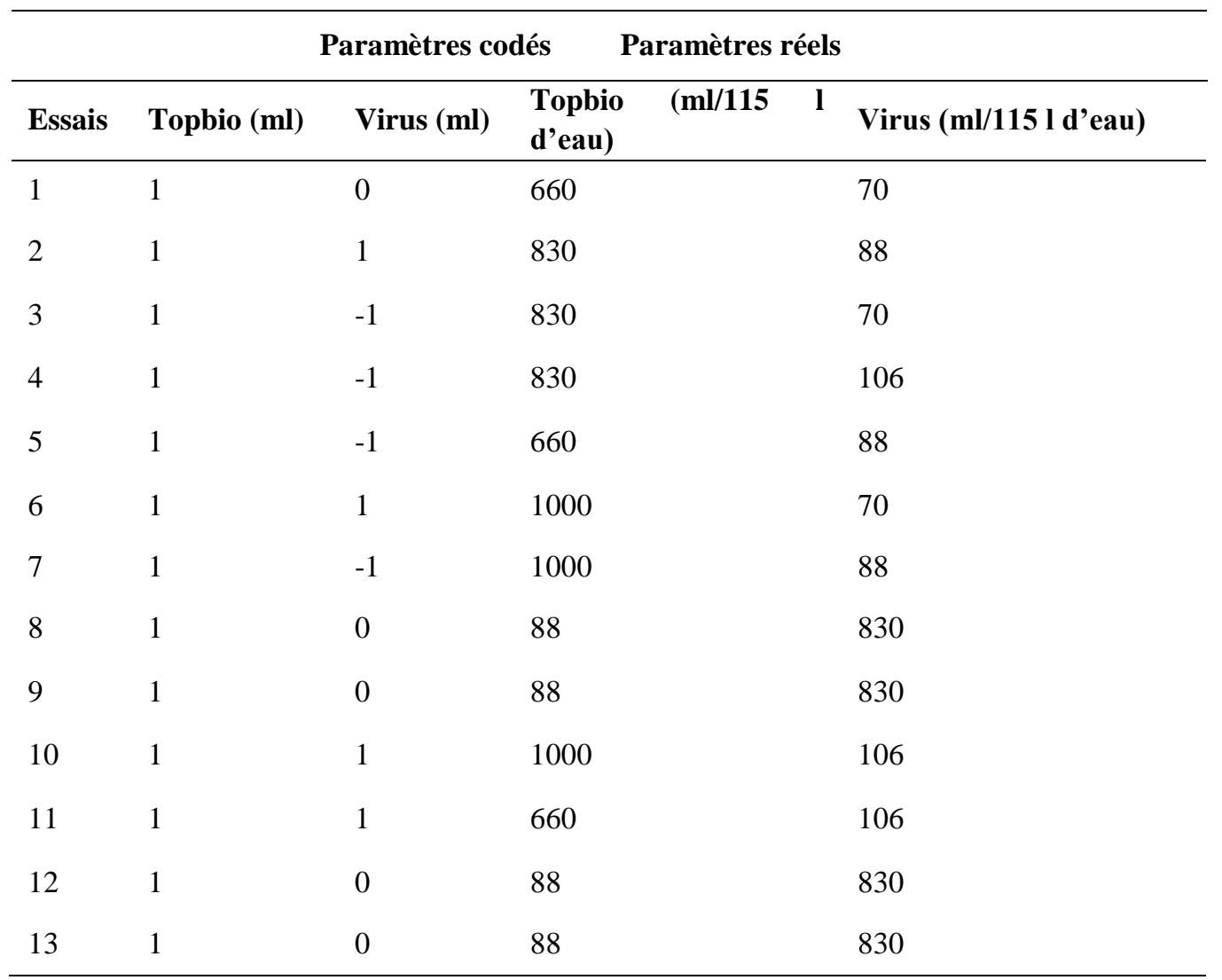




\section{RESULTATS}

Effet des doses des combinaisons de biopesticides sur le taux de mortalité de Aphis craccivora

L'effet des doses des combinaisons des biopesticides sur la mortalité des pucerons est présenté par la Figure 1a et $\mathrm{b}$. Les résultats ont montré que les taux de mortalité des pucerons à 3 jours et à 9 jours après l'inoculation sont influencés par les effets linéaires des doses de Topbio. Les taux de mortalité variaient de $30 \%$ à $38,33 \%$ et de $88,33 \%$ à $90,25 \%$ respectivement pour les pucerons à trois jours après l'inoculation et à neuf jours après l'inoculation (Tableau 2). On note que la croissance du taux de mortalité des pucerons s'accompagne d'une augmentation de la dose de Topbio (Figure 1a et 1b).

Effet des doses des combinaisons de biopesticides sur le taux de mortalité de Megalurothrips sjostedti

L'effet des combinaisons de biopesticides est très significatif $(\mathrm{p}<5 \%)$ sur les thrips. L'analyse du Tableau 2 indique que les taux de mortalité de Megalurothrips sjostedti à trois jours après inoculation et neuf jours après inoculation ont varié respectivement de $31,66 \%$ à $38,33 \%$ et de $86,66 \%$ à $95 \%$. Les effets linéaires et quadratiques des doses de Topbio et de virus MaviMNPV ont significativement $\quad(\mathrm{p}<0,05) \quad$ influencé la mortalité des thrips. La croissance des taux de mortalité des thrips est proportionnelle à l'augmentation de la dose de Topbio (Figure 2a et b)

Effet des doses des combinaisons de biopesticides sur le taux de mortalité des larves de Maruca vitrata

L'influence de la combinaison des doses de Topbio et du virus MaviMNPV sur le taux de mortalité des larves de Maruca vitrata à trois et neuf jours après inoculation est présentée respectivement par la Figure $3 a$ et $b$. L'analyse de la variance (ANOVA) du modèle de régression montre que les termes linéaires et quadratiques des doses de biopesticides (Topbio + virus MaviMNPV) ainsi que leur interaction ont un effet synergique très significatif $(\mathrm{p}<0,05)$ sur la mortalité des larves de Maruca vitrata. Ce taux varie de $25 \%$ à $55 \%$ et de $63,33 \%$ respectivement à trois et neuf jours après l'inoculation (Tableau 3 ).

Effet des doses des combinaisons de biopesticides sur le taux d'émergence et de mortalité des chrysalides de Maruca vitrata en fonction du traitement

Le taux d'émergence des larves de Maruca vitrata en fonction des doses de Topbio et du virus MaviMNPV a varié de $12,5 \%$ à $37 \%$ (Figure $4 a$ ). On note que l'augmentation de la dose de Topbio et du virus MaviMNPV entraîne une diminution du taux d'émergence des larves. L'inoculation des insectes avec des combinaisons de Topbio et virus MaviMNPV a permis d'obtenir des taux de mortalité des chrysalides compris entre $44,44 \%$ et $87,5 \%$. On remarque que le taux de mortalité des chrysalides croissait significativement avec l'augmentation de la dose du virus. Mais pour la dose de Topbio, la différence n'est pas significative (Figure 4b).

Effet des doses des combinaisons de biopesticides sur le taux d'émergence et de mortalité des chrysalides de Maruca vitrata en fonction du temps.

Les taux d'émergence et de mortalité des chrysalides de Maruca vitrata en fonction du temps sont respectivement présentés par les Figures 5 et 6 . L'analyse montre qu'au niveau de tous les traitements, le taux d'émergence évolue de façon croissante à partir du $5^{\text {ième jour }}$ après inoculation jusqu'au $8^{\text {ième jour après }}$ inoculation, avant de chuter au $9^{\text {ième jour après }}$ inoculation. Quant au taux de mortalité des chrysalides, il évolue inversement au taux d'émergence des larves de Maruca vitrata. La mortalité des chrysalides a commencé au $5^{\text {ième }}$ jour après inoculation, et décroit jusqu'au $9^{\text {ième }}$ jour après inoculation.

\section{Optimisation des doses des biopesticides}

Les doses optimales de Topbio et virus MaviMNPV ont été déterminées en indiquant l'objectif visé pour chaque réponse. Les valeurs des fonctions de désirabilité $(d)$ sont comprises entre 0 et 1 . La valeur de 0 est 
attribuée lorsque les facteurs conduisent à une réponse inacceptable (non désirable) et celle de 1 lorsque la réponse représente la performance maximale désirée pour les facteurs considérés. Les valeurs ont été fixées de manière à maximiser les taux de mortalité des insectes et à minimiser les taux d'émergence des larves d'insectes. Les résultats ont révélé que pour une meilleure efficacité des biopesticides, les doses de Topbio et du virus MaviMNPV seraient respectivement $1000 \mathrm{ml}$ et $106 \mathrm{ml}$, avec un taux de désirabilité de $85 \%$.

Tableau 2 : Valeurs expérimentales du plan composite centré.

\begin{tabular}{ccccccccc}
\hline Essais & Y1 & Y2 & Y3 & Y4 & Y5 & Y6 & Y7 & Y8 \\
\hline 1 & 25 & 73,33 & 33,33 & 90 & 30 & 90 & 35 & 87,5 \\
2 & 40 & 63,33 & 31,66 & 88,33 & 38,33 & 88,33 & 35 & 63,63 \\
3 & 28,33 & 85 & 36,66 & 86,66 & 43,33 & 90 & 12,5 & 44,44 \\
4 & 48,33 & 73,33 & 35 & 95 & 40 & 95 & 25 & 62,5 \\
5 & 38,33 & 81,66 & 38,33 & 88,33 & 35 & 88,33 & 17,5 & 63,63 \\
6 & 35 & 78,33 & 36,66 & 90 & 36,66 & 91,66 & 27,5 & 84,61 \\
7 & 45 & 71,66 & 35 & 85 & 43,33 & 91,66 & 27,5 & 64,7 \\
8 & 40 & 63,33 & 31,66 & 88,33 & 38,33 & 88,33 & 35 & 63,63 \\
9 & 40 & 63,33 & 31,66 & 88,33 & 38,33 & 88,33 & 35 & 63,63 \\
10 & 55 & 65 & 38,33 & 91,66 & 46,66 & 91,66 & 30 & 57,14 \\
11 & 40 & 63,33 & 31,66 & 88,33 & 38,33 & 88,33 & 35 & 63,63 \\
12 & 40 & 63,33 & 31,66 & 88,33 & 38,33 & 88,33 & 35 & 63,63 \\
13 & 41,66 & 70 & 35 & 95 & 31,66 & 93,33 & 37,5 & 83,33 \\
\hline
\end{tabular}

Y1 : taux de mortalité des larves de Maruca vitrata à 3 jours après l'inoculation ; Y2 : taux de mortalité des larves de Maruca vitrata à 9 jours après l'inoculation ; $Y 3$ : taux de mortalité des Megalurothrips sjostedti à 3 jours après l'inoculation ; Y4 : taux de mortalité des Megalurothrips sjostedti à 9 jours après l'inoculation ; Y5 : taux de mortalité des Aphis craccivora à 3 jours après l'inoculation ; $Y 6$ : taux de mortalité des Aphis craccivora à 3 jours après l'inoculation ; Y7 : Taux d'émergence des larves de Maruca vitrata ; Y8: Taux de mortalité des chrysalides émergés.

Tableau 3 : Analyse de la variance de l'efficacité des biopesticides.

\begin{tabular}{cccccccccc}
\hline Source & DF & Y1 & Y2 & Y3 & Y4 & Y5 & Y6 & Y7 & Y8 \\
\hline Régression & 5 & 694,06 & 297,07 & 29,42 & 31,83 & 137,88 & 23,45 & 99,86 & 366,23 \\
Linéaire & 2 & 671,33 & 206,01 & 7,87 & 11,56 & 128,13 & 13,38 & 38,54 & 196,10 \\
Carré & 2 & 16,48 & 88,28 & 18,75 & 17,49 & 9,05 & 9,36 & 59,75 & 166,89 \\
Interaction & 1 & 6,250 & 2,77 & 2,78 & 2,77 & 0,69 & 0,69 & 1,56 & 3,24 \\
Erreur Résiduelle & 7 & 16,19 & 403,75 & 51,42 & 70,35 & 112,04 & 36,80 & 605,90 & 1328,73 \\
Lack-of-Fit & 3 & 13,98 & 368,16 & 42,49 & 34,76 & 76,45 & 16,80 & 600,90 & 1018,26 \\
Erreur Pure & 4 & 2,204 & 35,59 & 8,92 & 35,59 & 35,59 & 20,00 & 5,00 & 310,47 \\
Total & 12 & 710,25 & 700,83 & 80,84 & 102,19 & 249,93 & 60,25 & 705,76 & 1694,96 \\
\hline
\end{tabular}

$Y 1$ : taux de mortalité des larves de Maruca vitrata à 3 jours après l'inoculation ; Y2 : taux de mortalité des larves de Maruca vitrata à 9 jours après l'inoculation; Y3 : taux de mortalité des Megalurothrips sjostedti à 3 jours après l'inoculation ; Y4 : taux de mortalité des Megalurothrips sjostedti à 9 jours après l'inoculation ; Y5 : taux de mortalité des Aphis craccivora à 3 jours après l'inoculation ; Y6: taux de mortalité des Aphis craccivora à 3 jours après l'inoculation ; Y7 : Taux d'émergence des larves de Maruca vitrata ; Y8: Taux de mortalité des chrysalides émergés. 


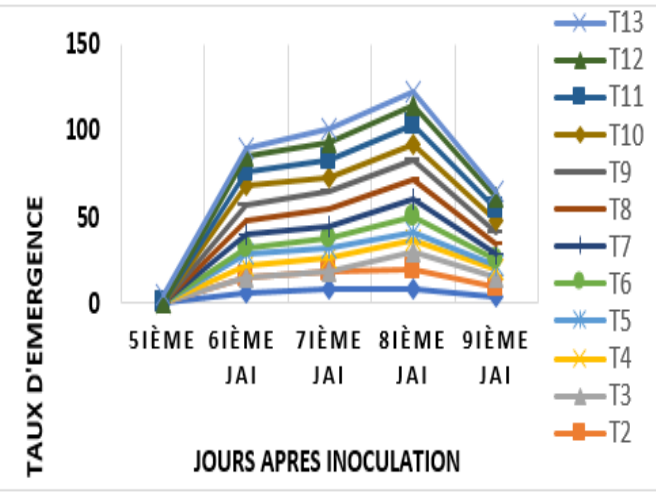

Figure 5 : Taux d'émergence des larves de Maruca vitrata.

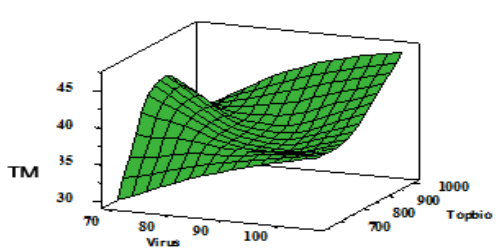

Figure 1a : Surface de réponse (3D) du taux de mortalité à $72 \mathrm{~h}$ après

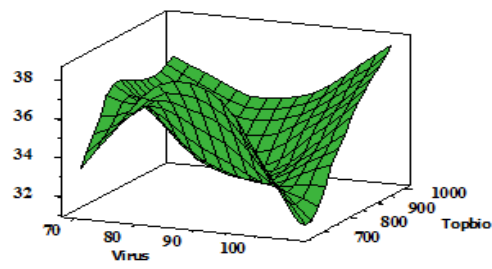

Figure 2a : Surface de réponse (3D) du taux de mortalité des thrips à $72 \mathrm{~h}$ après 1 'inoculation

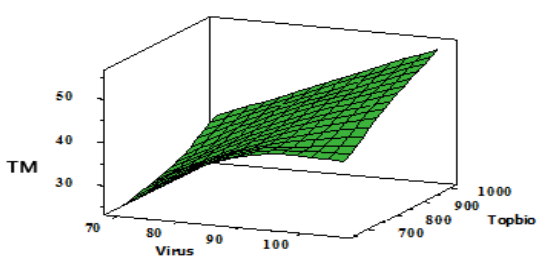

Figure 3a : Surface de réponse (3D) du taux de mortalité de Maruca vitrata à $72 \mathrm{~h}$ après 1 'inoculation

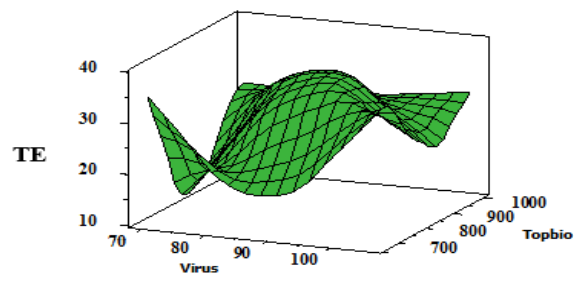

Figure 4a : Surface de réponse (3D) du taux d'émergence des larves Maruca

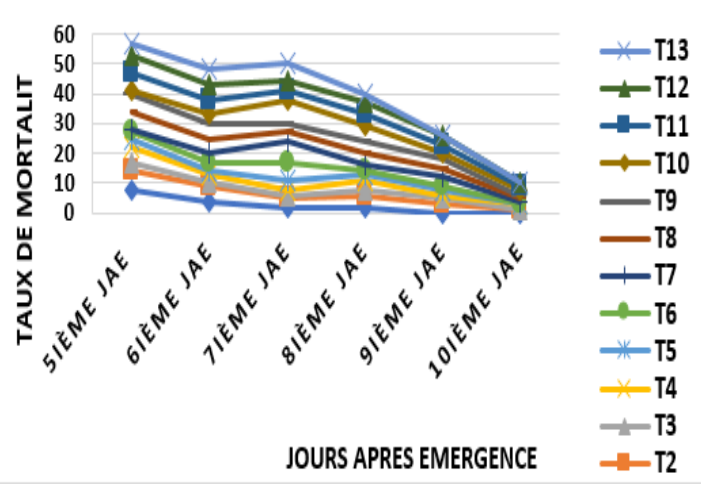

Figure 6 : Taux de mortalité des chrysalides de Maruca vitrata.

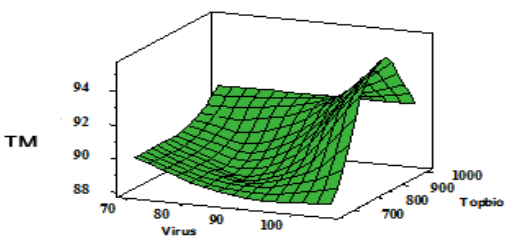

Figure 1b : Surface de réponse (3D) du taux de mortalité au 9 ième jour après

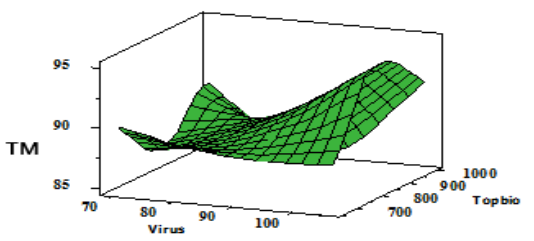

Figure 2b: Surface de réponse (3D) du taux de

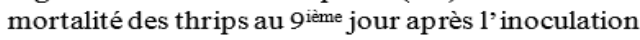

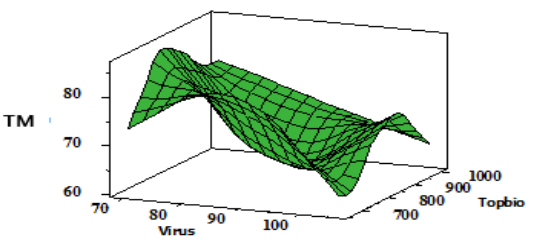

Figure 3b : Surface de réponse (3D) du taux de mortalité de Maruca vitrata au 9 ième jour après 1'inoculation

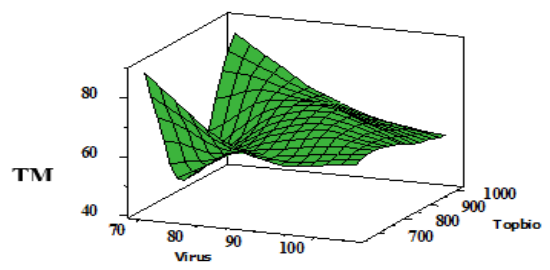

Figure 4b : Surface de réponse (3D) du taux de mortalité des chrysalides de Maruca vitrata 


\section{DISCUSSION}

Les biopesticides sont des agents potentiels de lutte biologique et constituent une composante importante au sein de systèmes de gestion intégrée des insectes ravageurs. Très développés à travers le monde ces dernières années, leur principe d'action repose sur l'action inhibitrice de la nutrition et de la croissance des insectes ravageurs de culture. Leur efficacité dans l'amélioration de la productivité et la maîtrise des insectes ravageurs dépendent de l'utilisation de la dose optimale des biopesticides. Dans la présente étude, les doses de la combinaison de deux biopesticides (Topbio et virus MaviMNPV) ont été optimisées par la méthode des surfaces de réponses. Les résultats ont montré que les populations de trois principaux ravageurs du niébé étaient significativement influencées par la variation des doses de ces biopesticides. Les pucerons (Aphis craccivora et Megalurothrips sjostedti) sont plus sensibles à une forte dose de Topbio qu'au virus MaviMNPV. Cela pourrait s'expliquer d'une part, par la spécificité d'action du virus MaviMNPV prononcée sur les larves de Maruca vitrata (Tamò et al., 2003), et d'autre part, par l'effet insecticide et anti-appétant de l'azadirachtine contenue dans Topbio (Gauvin et al., 2003). Les résultats obtenus dans cette étude sont en accord avec ceux rapportés par Traoré et al. (2019) qui ont montré qu'une réduction significative était observée dans la population des thrips (Megalurothrips sjostedti) après pulvérisation des champs expérimentaux avec le neem à des concentrations variant de 0,74 à 5\%, au Burkina Faso. L'effet insecticide de l'azadirachtine sur Aphis craccivora a été aussi rapporté par Muhammad et al. (2018) et Eziah et al. (2011) qui ont trouvé des taux de mortalité de $72,03 \%$ des jassides et de $55,00 \%$ des larves de Ephesia plutella respectivement après application de $5 \%$ d'huile de neem et après 4 jours d'exposition à une concentration de $6 \mathrm{ml} / \mathrm{l}$ d'huile de neem. Par ailleurs, il était observé que l'augmentation de la dose de l'huile de neem entraîne une forte réduction de la population des pucerons Aphis craccivora (Sokame et al., 2015). Des résultats similaires ont été rapportés par Elegbede (2015) et Camarda et al. (2018) qui ont obtenu respectivement une réduction au moins de 50\% et de $94,67 \%$ de la population de Helicoverpa armigera et de Dermanyssus gallinae), après pulvérisation de l'huile de neem à une dose de $15 \%$ et à $20 \%$ respectivement. Toutes ces données confirment l'efficacité de l'azadirachtine contenue dans Topbio, sur les ravageurs de culture, dont les thrips et les pucerons qui sont des ravageurs floricoles du niébé.

La pathogénicité de Topbio et du baculovirus MaviMNPV sur les différents stades de développement de Maruca vitrata a été étudiée à travers les taux d'émergence des larves et de mortalité des chrysalides. L'analyse des résultats a montré que l'effet du traitement est très significatif $(\mathrm{p}<5 \%)$ sur les larves et les chrysalides de Maruca vitrata. Le taux d'émergence des larves diminue en fonction de l'augmentation de la dose du virus et de Topbio et pourrait s'expliquer par le fait que le baculovirus Maruca vitrata Multi Nucleopolyhedrovirus (MaviMNPV) s'attaque à tous les stades de développement des larves de Maruca vitrata et induit des mortalités larvaires élevées (Tamò et al., 2003). Cette diminution serait également liée d'une part à l'effet inhibiteur de croissance des larves de Maruca vitrata associé à Topbio et d'autre part, au nombre de virions contenus dans chaque dose de virus. En effet, le principal composé actif de Topbio (l'azadirachtine) qui est un triterpénoïde, agit comme régulateurs de croissance des larves (Sanou, 2018), inhibiteur de l'oviposition (Faye, 2010) chez un grand nombre de ravageurs et inhibiteur de la nutrition chez les insectes (Nukenine et al., 2011). Ces résultats sont en accord avec ceux de Mama et al. (2016) qui ont observé des taux d'émergence allant jusqu'à $100 \%$ après application de $15 \%$ d'huile de neem sur les larves de Maruca vitrata aux stades L2, L3 et L4. Par ailleurs, l'augmentation du taux de mortalité des chrysalides en fonction de la dose du virus MaviMNPV pourrait être due au fait que les larves seraient infectées depuis le stade L3 de leur développement, avant de devenir 
des chrysalides où elles sont mortes suite aux effets des virions. Ces observations confirment ceux de Schenk et al. (2001) qui ont montré que, lorsque les composantes du neem spécialement l'azadirachtine entrent dans le corps des larves, l'activité de l'ecdysome (enzyme contrôlant le passage de l'état de larve à l'état adulte) est interrompue. Ceci empêche la métamorphose chez ces dernières qui demeurent ainsi à l'état larvaire jusqu'à la mort.

Les effets de la combinaison de Topbio et $\mathrm{du}$ virus MaviMNPV sur les taux d'émergence des larves et de mortalité des chrysalides ont également varié en fonction du temps. Les différences observées en fonction du temps, seraient liées à la biologie de Maruca vitrata qui comporte cinq stades larvaires (L1; L2 ; L3 ; L4 ; L5) avant la formation des chrysalides et à l'effet des virions contenus dans le virus MaviMNPV qui, après avoir été ingérés par les larves L3, n'auraient pas pu liquéfier la paroi des quelques larves survivantes avant le passage de ces dernières à l'étape de la chrysalidation. En effet, avant la chrysalidation (passage du stade larvaire au stade de chrysalides ou formation des chrysalides), il y a une période de 1 à 2 jours au cours de laquelle la larve devient verdâtre, perd tous ses autres pigments et cesse de s'alimenter (Song-Tay et al., 2007 ; Houihouigan, 2014). Ces résultats confirment ceux de Lee et al. (2007) qui rapportent que, les jeunes stades larvaires de Maruca vitrata développent une grande sensibilité au virus, comparativement aux stades proches de la chrysalidation. Par ailleurs, la baisse du taux d'émergence observée en fonction du temps pourrait être liée à l'effet inhibiteur de croissance des larves de Maruca vitrata associé à l'azadirachtine contenu dans Topbio (Mehinto et al., 2015). Les travaux de Bruce et al. (2004) ont montré que l'effet de l'huile de neem dure jusqu'au moins 10 jours sur Sesamia calamistis et Eldana saccharina.

En définitive, les résultats des modèles statistiques indiquent que les doses optimales de Topbio et du virus à utiliser pour l'amélioration de la productivité et la maitrise des insectes ravageurs étaient respectivement de $1000 \mathrm{ml}$ et de $106 \mathrm{ml}$ avec un taux de désirabilité de $85 \%$.

\section{Conclusion}

La pression des insectes ravageurs constitue la principale contrainte de culture du niébé en Afrique subsaharienne. Dans le but de mieux maitriser ces insectes ravageurs, il est possible de combiner les biopesticides pour une meilleure efficacité. Les courbes de surfaces des taux de mortalité des ravageurs étaient étudiées et les doses optimales de 1000 $\mathrm{ml}$ et de $106 \mathrm{ml}$ respectivement pour topbio et virus MaviMNPV avec $85 \%$ comme taux de désirabilité étaient obtenues. Ces biopesticides bien qu'étant efficaces dans les conditions de laboratoire, méritent d'être évalués en milieu réel, en vue de leur utilisation comme un produit alternatif aux insecticides chimiques dans la lutte contre les insectes ravageurs du niébé. Ainsi, ils garantiront la production des aliments de qualité répondant aux besoins des consommateurs.

\section{CONFLIT D'INTERETS}

Il n'y a aucun conflit d'intérêts entre les auteurs de cet article.

\section{CONTRIBUTIONS DES AUTEURS}

AGS et KA ont conçu le protocole ; MSPN a contribué à la rédaction du manuscrit. KOC et MMS ont supervisé l'étude. Les auteurs ont lu et approuvé la version finale du manuscrit.

\section{REMERCIEMENTS}

Les auteurs du présent article remercient l'Association de Recherche Ouest Africaine (West African Research Association: WARA) et la Banque Mondiale pour le soutien financier accordé au premier auteur de cet article. Les auteurs remercient également les examinateurs pour leurs contributions à l'amélioration du manuscrit.

\section{REFERENCES}

Abdoulaye O, Baoua I, Boureima S, Amadou L, Tamo M, Mahamane S, Mahamane A, 
Pittendrigh BR. 2018. Etude de l'efficacité des biopesticides dérivés du Neem et de l'entomopathogene MaviNPV pour la gestion des insectes ravageurs du niébé au Niger. Bulletin de la Recherche Agronomique du Bénin, 83: 16-24.

Abdourahamane Harouna M, Baoua I, Lawali S, Tamò M, Amadou L, Mahamane S, Pittendrigh B. 2019. Essai comparatif de l'utilisation des extraits du Neem et du virus entomopathogène MaviNPV dans la gestion des insectes ravageurs du niébé en milieu paysan au Niger. Int. J. Biol. Chem. Sci., 13(2): 950-961. DOI: https://dx.doi.org/10.4314/ijbcs.v13i2.30

Appert J, Deuse J. 1982. Les Ravageurs des Cultures Vivrières et Maraîchères sous les Tropiques. Moissonneuse et Larose : paris-France.

Ben R, Sue EH, Jenny SC, Rosemary SH. 2005. The role of plant and pathogeninduced behaviour in the persistence of a nucleopolyhedrovirus. Journal of Invertebrate Pathology, 88: 49-57. DOI: 10.1016/j.jip.2004.09.005

Boukar O, Belko N, Chamarthi S, Togola A, Batieno J, Owusu E, Haruna M, Diallo S, Umar ML, Olufajo O, Fatokun C. 2019. Cowpea (Vigna unguiculata): Genetics, genomics and breeding. Plant Breeding, 138: 415-424.

Bruce YA, Gounou S, Chabi-Olaye A, Smith H, Schulthess F. 2004. The effect of neem (Azadirachta indica A. Juss) oil on oviposition, development and reproductive potentials of Sesamia calamistis Hampson (Lepidoptera: Noctuidae) and Eldana saccharina Walker (Lepidoptera: pyralidae). Agricultural and Forest Entomology, 6: 1-10.

Camarda A, Pugliese N, Bevilacqua A, Circella E, Gradoni L, George D, Sparagano O, Giangaspero A. 2018. Efficacy of a novel neem oil formulation $\left(\mathrm{RP} 03^{\mathrm{TM}}\right)$ to control the poultry red mite Dermanyssus gallinae. Medical and Veterinary Entomology, 32: 290-297.
Dent DR. 1991. Insect Pest Management (éd). CAB International: UK.

Egho EO. 2011. Evaluation of Neem Seed Extract for the Control of Major Field Pests of Cowpea (Vigna unguiculata L. Walp) under Calendar and Monitored Sprays. Advances in Environmental Biology, 5(1): 61-66. DOI: http://dx.doi.org/10.5539/jas.v4n1p181

Elegbede M. 2015. Les ravageurs associés à la production cotonnière au Bénin : perceptions des producteurs, capacité de défense du cotonnier (Gossypium hirsutum) et potentialité de deux pesticides botaniques à base de neem (Azadirachta indica A Juss) dans le contrôle de Helicoverpa armigera Hûbner et Aphis gossypii Glover. Thèse de Doctorat d'Etat, Université d'Abomey-Calavi, Bénin, 176p.

El-Jasser HCA. 2011. Chemical and biologie properties of local cowpea seed protein seed protein grown in Gisan Region. International Journal of Biological, Biomolecular, Agricultural, Food and Biotechnological Engineering, 5(8): 466472.

DOI: https://doi.org/10.5281/zenodo.1078332

Ekesi S. 1999. Insecticide resistance in field populations wasp of the legume podborer, Maruca vitrata Fabricius (Lepidoptera: Pyralidae), on cowpea, Vigna unguiculata (L.) Walp. in Nigeria. International Journal of Pest Management, 45: 57-59. DOI: https://doi.org/10.1080/09670879922805 8

Eziah VY, Sackey I, Boateng BA, Obeng-Ofori D. 2011. Bioefficacy of neem oil $\left(\right.$ Calneem $\left.{ }^{\mathrm{TM}}\right)$, a botanical insecticide against the tropical warehouse moth, Ephestia cautella. Agricultural Science and Soil Science, 1(7): 242-248.

Faye M. 2010. Nouveau procédé de fractionnement de la graine de neem (Azadirachta indica A. Juss.) sénégalais : production d'un biopesticide d'huile et de tourteau. Thèse de Doctorat, Université de Toulouse, France, 267p. 
Gauvin MJ, Bélanger A, Nébié R, Boivin G. 2003. Azadirachta indica: l'azadirachtine est-elle le seul ingrédient actif? Phytoprotection, 84(2): 115-119. DOI: 10.7202/007814ar

Hounhouigan AD, Kossou D, Zannou E, Tamò M. 2016. Test de dosages et de combinaison des biopesticides pour la gestion des principaux ravageurs du niébé (Vigna unguiculata) et effet résiduel du virus MaviMNPV sur le niébé après la récolte. Mémoire de master, Faculté des sciences agronomiques et de l'environnement, Université Catholique de l'Afrique de l'Ouest, Benin, pp. 26-30.

Jimaja A, Mbang A, Mounjouenpou P, Mahob RJ, Mbarga AM, Mouen BJ, Nyasse SD, Nomo LBL, Tchouamo IR, Babin R. 2012. Evaluation naturelle de l'impact de Beauveria bassiana : champignon enthomopathogène dans la dynamique de population de Hypothenemus hampei, scolyte de baies des cerises de Coffea canefora. African Crop Science Journal, 20(s2): 443-451.

Kadri A, Zakari Moussa O, Sidoyacouba A, Hame Abdou KK, Karimoune L. 2013. Gestion intégrée de Maruca vitrata (FABRICIUS, 1787) et Megalurothrips sjostedti (TRYBOM, 1908), deux insectes ravageurs majeurs du niébé au Niger. Int. J. Biol. Chem. Sci., 7(6): 25492557.

DOI:

http://dx.doi.org/10.4314/ijbcs.v7i6.29

Kumar P, Lu-Ying ZH, Srinivasan R. 2014. Effect of three commercial biopesticides of neem (Azadirachta indica) and Bacillus thuringiensis on legume pod borer (Maruca vitrata) (Lepidoptera: Crambidae) in Thailand. International Journal of Tropical Insect Science, 34(2): 80-87.

Kek SP, Chin NL, Yusof YA. 2014. Simultaneous time-temperature-thickness superposition theoretical and statistical modelling of convective drying of guava. Journal of Food Science and Technology, 51(12): 3609-3622.
Kindozandji A, Kpindou OKD, Amevoin K, Nondichao AN, Glitho AI, Tamo M. 2020. Evaluation au laboratoire de l'efficacité de la combinaison de l'huile de neem et du champignon entomopathogène Beauveria bassiana vis-à-vis de Maruca vitrata (Fabricius) (Lepidoptera : Crambidae). Science de la Vie, de la Terre, et Agronomie: Rev. Ramres, 08(1): 2424-7235.

Lee ST, Srinivasan R, Wu YJ, Talekar NS. 2007. Occurrence and characterization of a nucleoplyhedrovirus from Maruca vitrata (Lepidoptera: Pyralidae) in Taiwan. Biological Control, 52 : 801-819.

Mama BE, Agbaka A, Tamò M. 2016. Evaluation de l'efficacité de l'huile de neem, biovirus Mavi MNPV et leur combinaison dans le contrôle des larves de Maruca vitrata au laboratoire. Mémoire de licence, Génie de l'environnement, Université d'Abomeycalavi, Benin, pp. 29-32.

Mehinto JT, Atachi P, Elégbédé M, Douro Kpindou OK, Tamò M. 2014. Efficacité comparée des insecticides biologiques et chimiques dans la gestion des insectes ravageurs du niébé (Vigna unguiculata) au Bénin. Journal of Applied Biosciences, 84: $\quad$ 7674-7681. DOI: https://doi.org/10.4314/jab.v84i1.1

Mikpon A. 2017. Evaluation des effets de différentes doses de formulations de MaviMNPV virus et la combinaison avec l'huile de neem contre Maruca vitrata Fabricius (Lepidoptera: Crambidae), ravageur du niébé. Rapport de licence, Génie de l'environnement, Université d'Abomey-calavi, Benin, pp. 26-27.

Muhammad UA, Raza M, Waseem A, Mubasshir S, Javed AT, Muhammad I. 2018. Comparative efficacy of Neem derivatives and imidacloprid against some cotton pests. Journal of Entomology and Zoology Studies, 6(3): 113-117.

Nukenine EN, Tchiegang C, Mekouo AAT, Tofel KH, Adarkwah C, Obeng Ofori D, Adler C. 2011. Efficacy of Calneem derived from Ghanaian neem seeds and 
seed oils from two locations in Cameroon against Sitophilus zeamais (Coleoptera: Curculionidae) on maize. International Journal of Tropical Insect Science, 31(4): 225-234.

Ouédraogo E. 2004. L'utilisation des insecticides naturels dans la protection des cultures au Burkina Faso. Communication fait au CTR de Proceedings of the International Congress of Entomology 2008, Durban, South Africa. p.

621.

http://www.ice2008.org.za/pdf/proceedin gs.pdf.

Sanou KF, Ouédraogo S, Nacro S, Ouédraogo M, Kaboré-Zoungrana C. 2015. Durabilité de l'offre et valeur nutritive des fourrages commercialisés en zone urbaine de Bobo-Dioulasso, Burkina Faso. Cahiers Agricultures, 25(15002): 1-10.

Sokame BM, Tounou AK, Datinon B, Dannon EA, Agboton C, Srinivasan R, Pittendrigh BR, Tamò M. 2015. Combined activity of Maruca vitrata multinucleopolyhedrovirus, MaviMNPV, and oil from neem, Azadirachta indica Juss and Jatropha curcas L., for the control of cowpea pests. Crop Protection, 72: 150157.

DOI: https://doi.org/10.1016/j.cropro.2015.03. 016.

Song-Tay L, Srinivasan R, Yan-Jheng W. 2007. Occurrence and characterization of a nucleopolyhedrovirus from Maruca vitrata (Lepidoptera, pyralidae) isolated in Taiwan. BioControl, 52: 801-819. DOI: $10.1007 / \mathrm{s} 10526-006-9049-\mathrm{x}$

Reddy NP, Khan APA, Uma DK, Victor SJ, Sharma HC. 2008. Assessment of the suitability of Tinopal as an enhancing adjuvant in formulations of the insect pathogenic fungi Beauveria bassiana
(Bals.) Vuillemin. Pest Management Science: Formerly Pesticide Science, 64(9): $\quad 909-915 . \quad$ DOI: https://doi.org/10.1002/ps.1581

Schenk P, Indorf A, Fluri P. 2001. Effet de l'huile de neem sur l'acarien varroa et sur les abeilles. Revue Suisse d'Apiculture, 98(3) : 114- 119.

Tamò M, Ekesi S, Maniania NK, Cherry A. 2003. Biological control, a Non behavious component of IPM for cowpea. In Biological Control in IPM System in Africa, Neuenschwander P, Borgemeister C, Langewald J (eds). CABI: RoyaumeUni; 295-306.

Traore F, Waongo A, Drabo E, Yamkoulga M, Dabire-Binso C, Sanon A. 2019. Effet des périodes d'épandage des huiles de neem (Azadirachta indica L.) sur les populations de Megalurothrips sjostedti Trybom et de Maruca vitrata Fabricius dans la culture de niébé. Int. J. Biol. Chem. Sci., 13(3): 1300-1307. DOI: https://dx.doi.org/10.4314/ijbcs.v13i3.7

Traoré F, Waongo A, Ba NM, Sanou A, Tamò M, Pittendrigh RB. 2019. Effects of Maruca vitrata multinucleopolyhedrovirus and neem oil, Azadirachta indica Juss on the eggs of the cowpea pod borer, Maruca vitrata Fabricius (Lepidoptera: Crambidae). International Journal of Tropical Insect Science International Journal of Tropical Insect Science, 39(1). DOI: 10.1007/s42690-019-00061-2

Valda CAS, Reginaldo B, Edmilson JM, Jorge BT. 2003. Susceptibility of Plutella xylostella (L.) (Lepidoptera: Plutellidae) to the Fungi Beauveria bassiana (Bals) Vuil. And Metarhizium anisopliae (Metsch.) Sorok. Neotropical Entomology, 32: 653- 658. 